Report of the Second Bi-monthly Virtual Meeting of the Iranian Society of Cardiac Surgeons: Investigation of Three Interesting Cases

\author{
Yaser Toloueitabar (iD) ${ }^{1}$, Mohammad Reza Mirzaaghayan (ii) ${ }^{2}$, Amir Nasser Jadbabaei (iD ${ }^{3}$ and Sanaz \\ Asadian (iD) ${ }^{1, *}$ \\ ${ }^{1}$ Rajaie Cardiovascular Medical and Research Center, Iran University of Medical Sciences, Tehran, Iran \\ ${ }^{2}$ Tehran Children Hospital, Tehran University of Medical Sciences, Tehran, Iran \\ ${ }^{3}$ Department of Cardiac Surgery, Masih Daneshvari Hospital, Shahid Beheshti University of Medical Sciences, Tehran Iran \\ "Corresponding author: Rajaie Cardiovascular Medical and Research Center, Iran University of Medical Sciences, Tehran, Iran. Email: asadian_s@yahoo.com
}

Received 2021 June 09; Accepted 2021 July 31.

\begin{abstract}
The Iranian society of cardiac surgeons (ISCS) has been holding a regular meeting every two months since 2005. This is the report of one of the mentioned meetings in which three interesting cardiac surgery cases were presented and discussed.

Keywords: Anomalous Left Coronary Artery Connected to the Pulmonary Artery (ALCAPA), Ventricular Septal Defect, Cardiopulmonary Bypass, Innominate Artery Pseudoaneurysm, Persistent Fifth Aortic Arch
\end{abstract}

\section{Purpose of Meeting}

In the bi-monthly meetings, the Iranian cardiac surgeons introduce their challenging cases to discover the best approach for surgical management of the patients. Moreover, interesting cases, rare conditions, and novel techniques in cardiac surgery are discussed. We herein report three interesting cases that were discussed in the meeting that was held in December 2020.

\section{Summary of Presented Findings}

\subsection{A Challenging Case of Surgical Ventricular Septal Defect Clo-} sure

A 10-month-old male infant presented with a largesized subaortic ventricular septal defect (VSD), a mediumsized patent ductus arteriosus (PDA), and increased pulmonary arterial pressure. He suffered a failure to thrive as well as poor feeding and was on oral medication.

Positive findings on transthoracic echocardiography (TTE) were a mild enlargement of the left atrium (LA), mild mitral regurgitation (MR), moderate tricuspid regurgitation (TR), right ventricular (RV) enlargement, mild RV dysfunction and a large subaortic VSD $(9 \mathrm{~mm})$. The left ventricular (LV) ejection fraction (EF) was 55\%. The same findings were seen in cardiac computed tomography angiography (CCTA).
In operation, the PDA was closed and the VSD was repaired through the right atrial approach utilizing a pericardial patch (autologous). Weaning of the patient from cardiopulmonary bypass was very difficult and with a high dose of inotropes.

Postoperative TTE revealed severe LV dysfunction, moderate MR, and severe pulmonary hypertension (PH). However, there were no RV dysfunction and residual VSD. In the next 2 postoperative days in the pediatric intensive care unit (PICU), the clinical status and echocardiographic findings of the patient did not change. CCTA was done seeking the cause of LV dysfunction and surprisingly it revealed the anomalous origin of the left coronary artery from the pulmonary artery (ALCAPA). The patient underwent reoperation and was placed on extracorporeal membrane oxygenation (ECMO) after the indicated anomalous artery was translocated to the aorta. In the PICU, his LV function improved during the next few days, but he expired due to sepsis after ten days.

The presenter then discussed the pathophysiology of ALCAPA and the causes of missing it in the presented case as is coming in the next paragraph:

ALCAPA is a rare congenital disorder (occurrence rate: 1 in 300000 live births). It is considered the most prevalent cause of myocardial infarction in children, with up to $90 \%$ mortality in early childhood if it is not treated. Extensive inter-coronary collaterals lead to a 10 - 15\% survival to adulthood (1). As the pulmonary vascular resis- 
tance drops after birth, myocardial perfusion diminishes. In ALCAPA, the progressive development of collaterals results in the coronary steal. Consequently, the oxygenated blood enters the right coronary artery and flows into the low-resistant pulmonary artery. Given the presence of less coronary steal and sufficient coronary perfusion to the myocardium due to PH, ALCAPA can be obscured in the setting of left to right shunt. The causes of severe LV dysfunction after operation might be due to lack of collateral formation because of pre-operative balanced physiology of the heart, improper cardioplegic solution administration (poor LV protection), and sudden decrease in pulmonary artery pressure. In the presented patient, the direction of the proximal segment of the left main coronary artery in conjunction with the respiratory motion artifacts resulted in the obscuring of the ALCAPA in the preoperative CCTA (Figure 1A). Moreover, the preoperative CCTA was performed with the non-electrocardiogram-gated protocol, which contributed to the misdiagnosis. However, in the postoperative electrocardiogram-gated CCTA, the anatomy was determined exactly (Figure 2B). This finding emphasizes the role of accurate preoperative anatomical and functional assessments applying echocardiography and electrocardiogram-gated CCTA (2).

A couple of questions were asked and also some interesting recommendations were given by the audiences as follows:

The use of CT angiography for diagnosis of VSD was challenged by some attendees and they believed that it should not be done routinely as a diagnostic tool in simple VSD cases. Based on the literature, echocardiography is the gold standard method for the diagnosis of VSD (3). In the presented case, cardiac CTA was done because the anatomy of the aortic arch could not be delineated in the pre-operative echocardiography.

The use of ECG-gated cardiac CTA was challenged by one of the audiences due to its high dose of radiation. Lee et al. impressed that the prospective electrocardiogramgated CCTA is associated with acceptable quality and radiation dose. Therefore, they proposed this technique as the best choice for cardiac anatomy evaluation in the pediatric population (4).

One of the audiences recommended that the presence of significant MR in a patient with VSD should draw the surgeon's attention to the anatomy of the coronary arteries.

Another interesting recommendation was that the surgeon should always pay attention to coronary sinus fluid return during cardioplegic solution administration and if its amount is lower than expected, disorders such as coronary artery fistula or ALCAPA should be investigated.

\subsection{A Rare Case of Blunt Innominate Artery Trauma}

The patient was a young female with a history of multiple trauma due to a car accident 15 days before admission to the cardiovascular center. She had a history of chest tube insertion in the right hemithorax on the first day of admission due to multiple trauma. Moreover, multiple operations had been carried out for her in the primary trauma center. After initial stabilization at the trauma center, the patient was referred to the Rajaie Heart Center due to abnormal findings on chest X-rays. On arrival, stable vital signs and a Glasgow coma scale (GCS) of 15 were registered. Peripheral pulses were symmetrical. Neurologic exams were unremarkable. A skin crush on the anterior aspect of the middle and right upper chest was noticed. On thoracic aorta CTA, a contrast-filled outpouching with mild border irregularity was detected in the ostioproximal part of the innominate artery that was compatible with the diagnosis of pseudoaneurysm (Figure 2).

The patient was transferred to the operating room. After administration of heparin, peripheral cannulation of the right femoral vein and artery was done and CPB started and deep hypothermia applied. After median sternotomy, the surgeon encountered with a very dense fibrosis of the upper mediastinum over the aortic arch and its branches and around the innominate vein. After releasing the adhesions and dissecting the mentioned structures, a pulsatile mass appeared. The most probable diagnosis was the pseudoaneurysm of proximal part of the innominate artery. After reaching the goal temperature, cardiac arrest and then total circulatory arrest (TCA) with antegrade cerebral perfusion started. A tear was noted on the superior aspect of the aortic arch which was extended to the origin of the left common carotid artery. Also, transection of the brachiocephalic artery in its origin was evident. Therefore, aortic arch was repaired and debranching of the innominate artery was done using an $8 \mathrm{~mm}$ Dacron tube graft. Endto-end anastomosis of the graft was performed proximally to the lateral aspect of ascending aorta and distally to the innominate artery. The patient weaned easily from CPB and had an eventless post-operative course and discharged from the hospital a few days after.

A couple of questions were asked by the audiences that comes in below:

The necessity of using TCA has been questioned by some audiences. The presenter mentioned the presence of very dense adhesions in the operating site and the inability to get an appropriate proximal control, the prudent reasons to apply TCA.

One of the audiences questioned if it was possible to use an endovascular approach to manage the patient. The applicability of the mentioned approach was challenged 

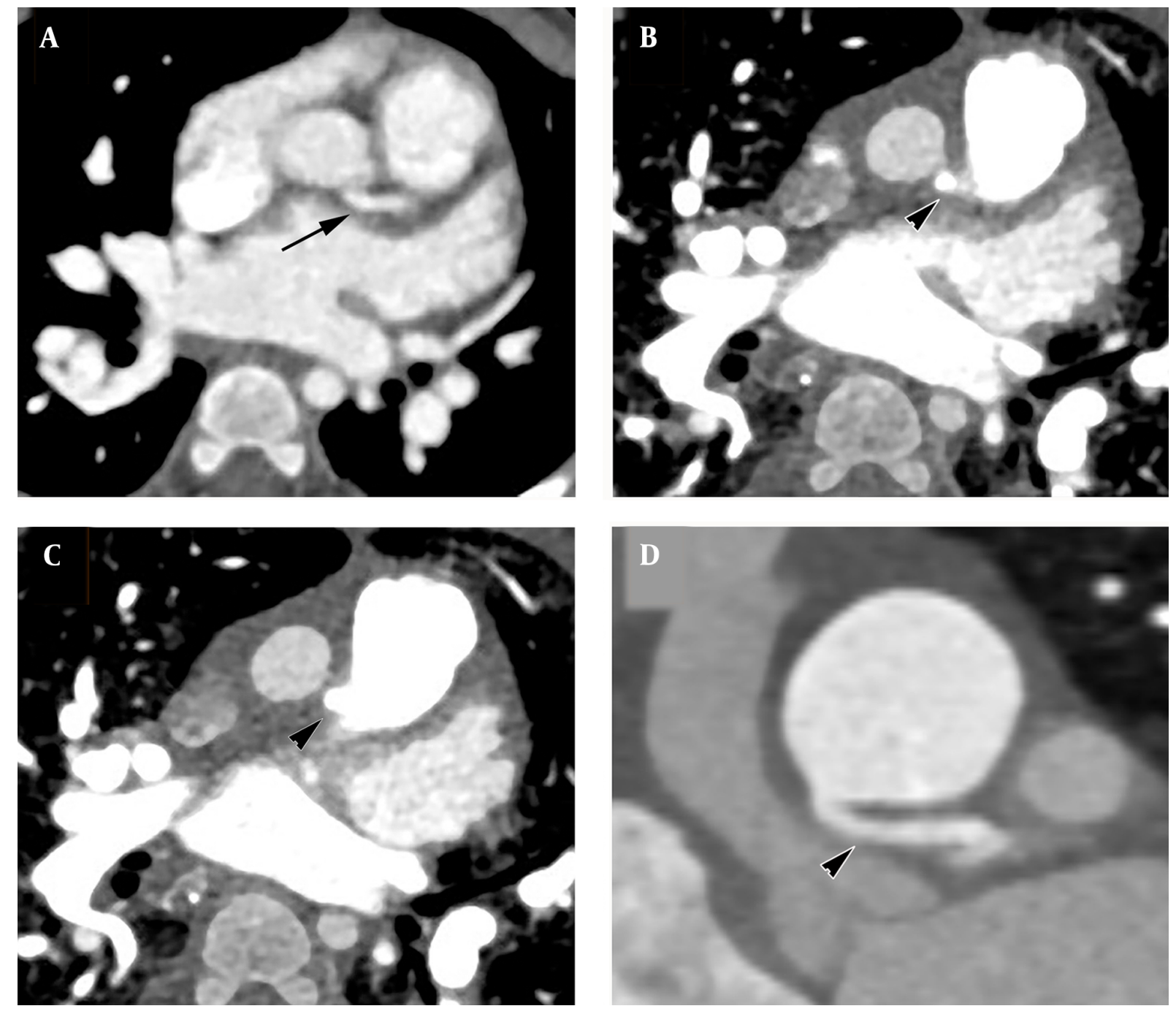

Figure 1. Cardiac CT angiography. A, non-electrocardiogram gated axial cardiac CT angiogram with the apparent origin of left main coronary artery from the left coronary cusp (arrow); B and C, axial; as well as D, oblique coronal; electrocardiogramgated pulmonary phase cardiac CT angiography depicts the anomalous origin of the left main coronary artery from the pulmonary artery (arrowheads).

by presenter because of lack of a good proximal landing zone.

Injury to the innominate artery is rare, however, it is fatal. This condition happens by different mechanisms, including blunt, penetrating, or iatrogenic trauma. Blunt trauma is the mechanism of injury in most cases rather than penetrating injury. Up to $70 \%$ of these injuries prove lethal at the scene with associated in-hospital mortality ranging from $10-70 \%$. The most commonly associated vascular and thoracic injuries are the carotid artery insults (22\%); pneumothorax (30\%); rib fractures (26\%) and tracheobronchial injuries (8\%) (5).

The most common mechanism of injury is motor- vehicle accidents. Head-on collisions constitute the majority at speeds of more than $60 \mathrm{mph}$ or change in speeds of greater than or equal to $20 \mathrm{mph}$ (6).

In blunt trauma of the innominate artery, the most commonly affected site is its origin from the aorta. According to the postmortem studies, motor vehicle-pedestrian accidents were associated with the highest percentage of significant vascular trauma (7).

Due to the anatomical location of branching site of the innominate artery from the aorta, there is minor incidence of pericardial effusion due to this injury itself. Therefore, injury of the ascending aorta must be kept in mind in the presence of pericardial effusion. 

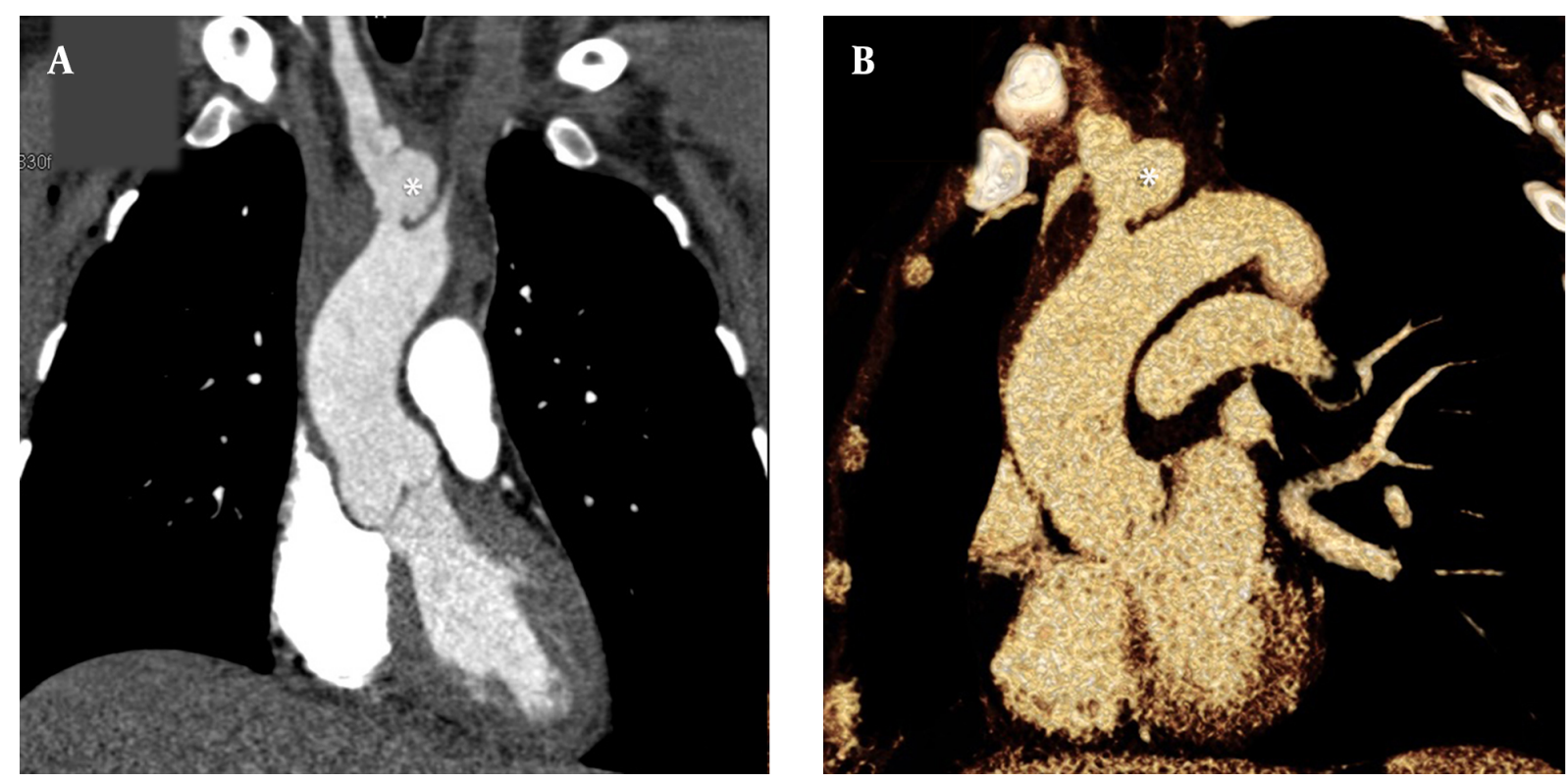

Figure 2. Thoracic CT angiography. A, Multiplanar reconstruction image; and B, volume-rendering technique; of great arteries in the coronal plane demonstrate a contrastfilled outpouching protruding from the proximal part of the innominate artery with irregular borders in favor of pseudoaneurysm (asterisks).

There are two treatment strategies for these patients; the endovascular repair and the open surgical repair. Indications for open repair include an unstable patient, a large rapidly expanding hematoma of the mediastinum or zone I of the neck, a penetrating injury to the chest, or failure of endovascular repair (5).

\subsection{A Three-Year-Old Girl with Nephrotic Syndrome and Hyper- tension}

A 3-year-old girl, known case of nephrotic syndrome and hypertension, who was under corticosteroid therapy and a high dose of anti-hypertensive drugs, underwent workup for the cause of hypertension. On TTE, coarctation of the aorta (COA) was diagnosed and the peak pressure gradient (PPG) at the site of stenosis was about $70 \mathrm{mmHg}$. Thoracic CT-angiography (CTA) revealed a large LA, severe concentric LV hypertrophy, and a discrete COA in the distal of the arch of aorta. Moreover, a comprehensive CTA evaluation revealed persistent fifth aortic arch (PFAA) (Figures $3 \mathrm{~A}$ and $\mathrm{B})$.

The patient became a candidate for surgical correction. The operation was done via a thoracotomy approach from the posterolateral aspect of the 4 th intercostal space. The PDA was closed and cut. The site of COA was resected and end-to-end anastomosis of the aorta was done (Figure 3C). The PPG was about $5 \mathrm{mmHg}$ at the level of anastomosis at the end of the operation. The patient had an eventless postoperative course and was discharged from the hospital a few days after.

The first case of PFFA was reported by Van Praagh in 1969 , as a case of "congenital double-lumen aortic arch" (8, 9). This rare anomaly has three subtypes: Type A or double lumen aortic arch is the most frequent type of PFAA in which the aortic arch divides into two lumens at the innominate artery level and again joins together in descending aorta level making one lumen. It differs from the double aortic arch that is a form of a vascular ring in which the trachea and esophagus pass through the limbs of two aortic arch while in the double lumen aortic arch, the trachea pass to the left or right of the aortic arch. In type $\mathrm{B}$, the interrupted superior lumen in association with the persistence of the inferior lumen is detected. Type $C$ is the condition of the existence of a systemic-to-pulmonary arterial connection. This type is usually accompanied by significant RV outflow stenosis or pulmonary atresia and VSD (Figure 4) (10).

Being a transient embryologic condition in most cases, the PFAA is often associated with a variety of anomalies including COA or interrupted aortic arch, as well as truncus arteriosus, pulmonary atresia, tricuspid atresia, and VSD (11).

The presenter confirmed that the endovascular intervention, recommended by one of the audiences, seems to be a proper choice for this patient. 

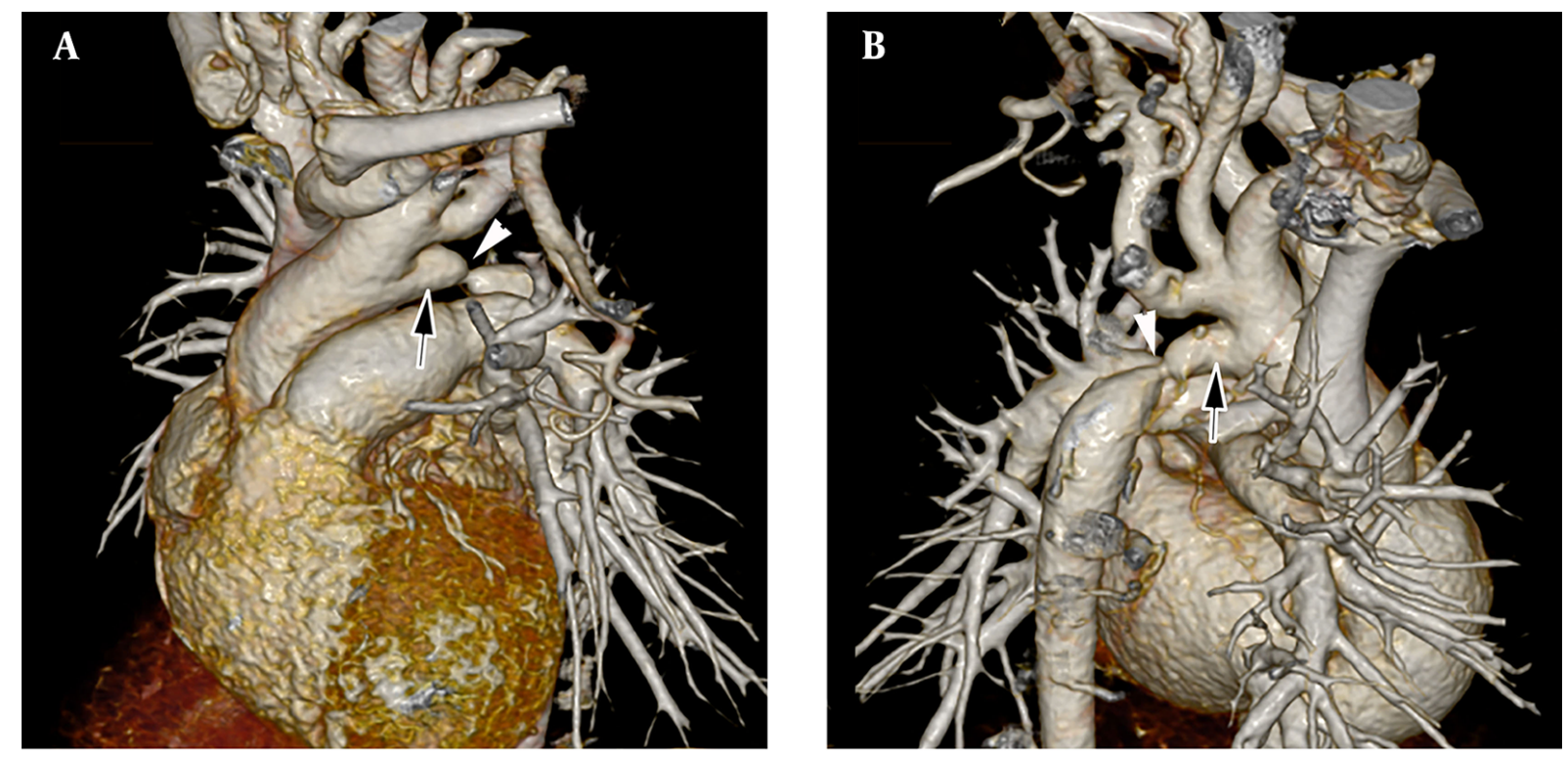

C
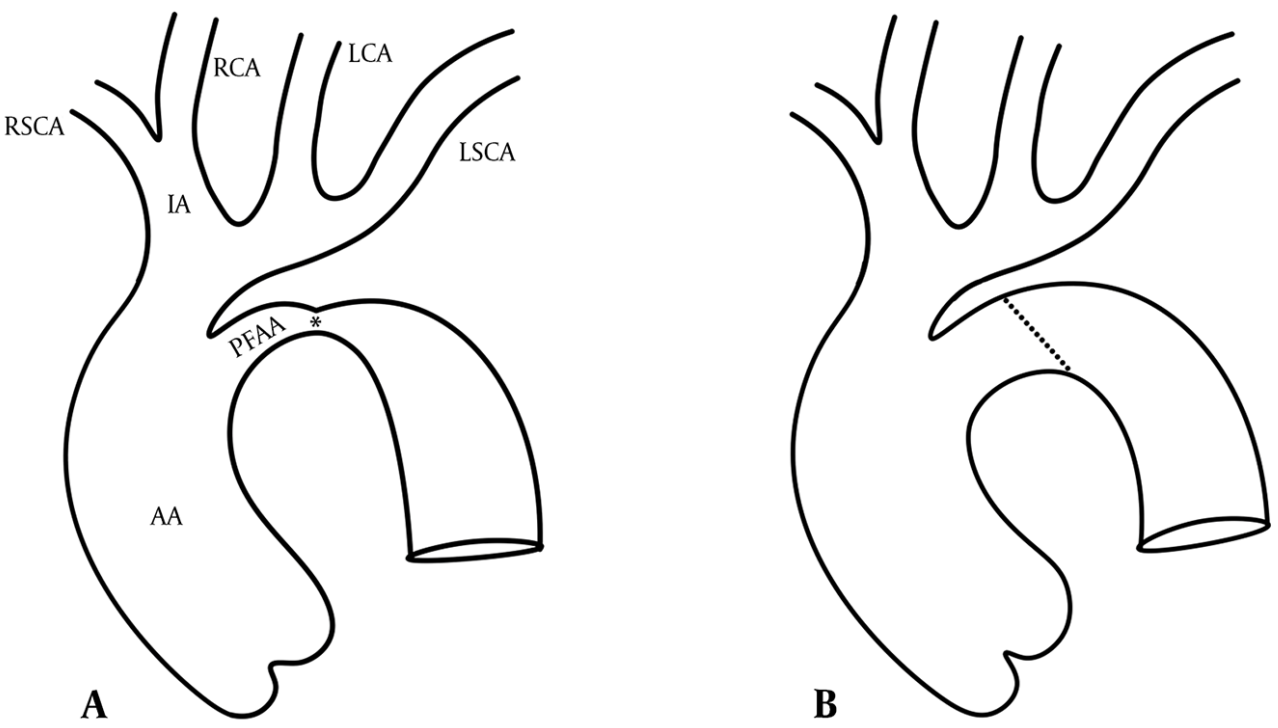

Figure 3. A and B, oblique-coronal volume-rendered reconstruction CT angiography images of the great arteries from A, anterior; and B, posterior views. Note the persistent fifth aortic arch (arrows) with associated coarctation (arrowheads); C, schematic illustrations of A, preoperative; and B, postoperative; configuration of the aortic arch (AA, ascending aorta; IA, innominate artery; R, right; L, left; SCA, subclavian artery; CA, carotid artery; PFAA, persistent fifth aortic arch).

\section{Recommendation for Future Research}

We suppose that some notable hints for further investigations are implied in the presented meeting. First, a retrospective investigation could be undertaken in congenital cases with $\mathrm{PH}$ secondary to a left-to-right shunt who undergo cardiopulmonary bypass to determine if the coronary anatomy is accurately evaluated or not preoperatively. As a result, we may assess our current condition and potentially alter institutional preoperative consideration methods.

Second, we could review medical and surgical records in a multicentric study for seeking cases of misdiagnosed PFAA in preoperative echocardiography, CCTA, catheterization, and cardiac MRI to investigate the role of multimodality imaging for the anatomic description and planning of this rare anomaly. 


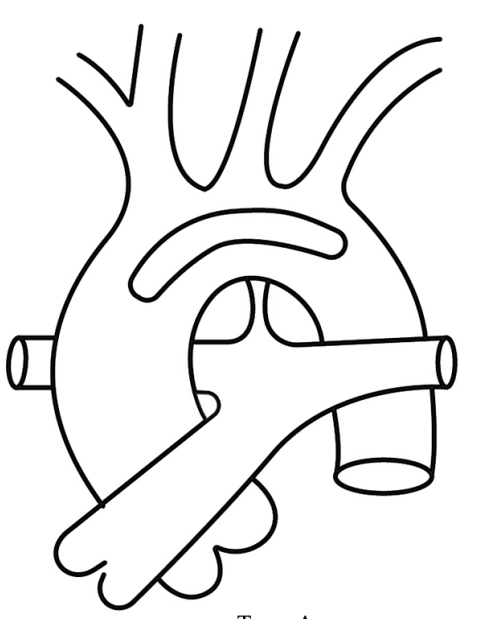

Type A

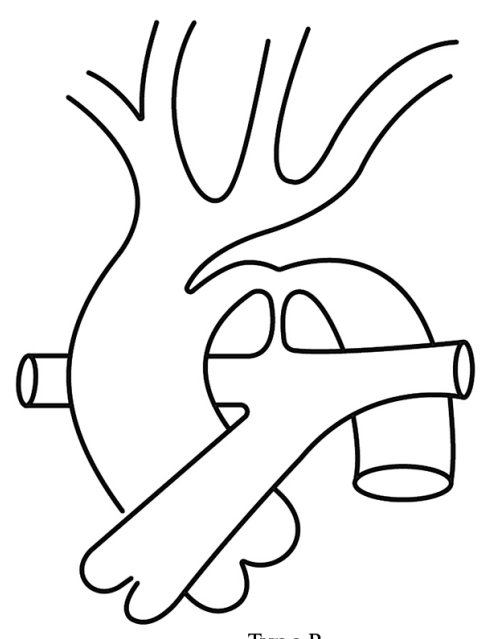

Type B

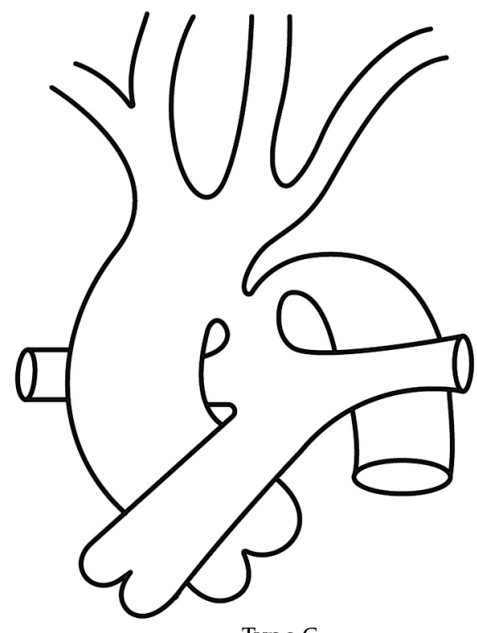

Type C

Figure 4. The classification of persistent fifth aortic arch described by Richard and Stella van Praagh.

\section{Acknowledgments}

We would like to thank the participants of this virtual meeting for their kind cooperation.

\section{Footnotes}

Authors' Contribution: Y. T, M.R.M, and A. N. J performed surgeries, presented the cases, and reviewed the manuscript. Y.T and S. A designed the manuscript, wrote the draft, and edited the final version. S. A submitted the manuscript.

Conflict of Interests: The authors declare no conflict of interest.

Funding/Support: No funding was achieved for this report.

\section{References}

1. Murala JS, Sankar MN, Agarwal R, Golla PN, Nayar PG, Cherian KM. Anomalous origin of left coronary artery from pulmonary artery in adults. Asian Cardiovasc Thorac Ann. 2006;14(1):38-42. doi: 10.1177/021849230601400110. [PubMed: 16432117].

2. Mahdavi M, Asadian S, Shahzadi H, Daliri M, Rezaeian N, Toloueitabar Y. Difficult weaning from cardiopulmonary bypass after surgical VSD closure: An unusual rare case. Clin Case Rep. 2021;9(1):1447. doi: 10.1002/ccr3.3486. [PubMed: 33489150]. [PubMed Central: PMC7813056].
3. Penny DJ, Vick GW. Ventricular septal defect. Lancet. 2011;377(9771):1103-12. doi: 10.1016/S0140-6736(10)61339-6. [PubMed: 21349577].

4. Lee YW, Yang CC, Mok GS, Wu TH. Infant cardiac CT angiography with 64-slice and 256-slice CT: Comparison of radiation dose and image quality using a pediatric phantom. PLoS One. 2012;7(11). e49609. doi: 10.1371/journal.pone.0049609. [PubMed: 23185380]. [PubMed Central: PMC3504147].

5. Bishop MA, Akbani MJ. Innominate artery injury. Treasure Island (FL): StatPearls Publishing; 2021.

6. Johnston RH, Wall MJ, Mattox KL. Innominate artery trauma: A thirty-year experience. J Vasc Surg. 1993;17(1):134-40. doi: 10.1067/mva.1993.42299. [PubMed: 8421329].

7. Hirose $\mathrm{H}$, Gill IS. Blunt injury of the innominate artery: A case report and review of literature. Ann Thorac Cardiovasc Surg. 2004;10(4):21823. [PubMed: 15458372].

8. Naimo PS, Vazquez-Alvarez Mdel C, d'Udekem Y, Jones B, Konstantinov IE. Double-lumen aortic arch: Persistence of the fifth aortic arch. Ann Thorac Surg. 2016;101(5):e155-6. doi: 10.1016/j.athoracsur.2015.10.014. [PubMed: 27106464].

9. Van Praagh R, Van Praagh S. Persistent fifth arterial arch in man. Congenital double-lumen aortic arch. Am J Cardiol.1969;24(2):279-82. doi: 10.1016/0002-9149(69)90417-2. [PubMed: 5799089].

10. Marcus BS, Rubio A, Deen JF. Transcatheter relief of coarctation of the aorta in a persistent fifth aortic arch anatomy. Prog Pediatr Cardiol. 2020;57:101200. doi: 10.1016/j.ppedcard.2020.101200.

11. Al Akhfash AA, Al Mutairi MB, Al Habshan FM. Persistent fifth aortic arch diagnosed by echocardiography and confirmed by angiography: Case report and literature review.J Saudi Heart Assoc. 2009;21(4):2458. doi: 10.1016/j.jsha.2009.10.003. [PubMed: 23960580]. [PubMed Central: PMC3727347]. 